\title{
Development of Penicillium italicum-Specific Primers for Rapid Detection among Fungal Isolates in Citrus ${ }^{\mathrm{E}}$
}

\author{
Kai Chen ${ }^{1,2}$, Zhonghuan Tian ${ }^{2}$, Fatang Jiang ${ }^{1}$, and Chao-an Long ${ }^{2 *}$ \\ ${ }^{1}$ School of Bioengineering and Food, Hubei University of Technology, Wuhan 430068, P.R. China \\ ${ }^{2}$ Key Laboratory of Horticultural Plant Biology of the Ministry of Education, National Centre of Citrus Breeding, Huazhong Agricultural \\ University, Wuhan 430070, P.R. China
}

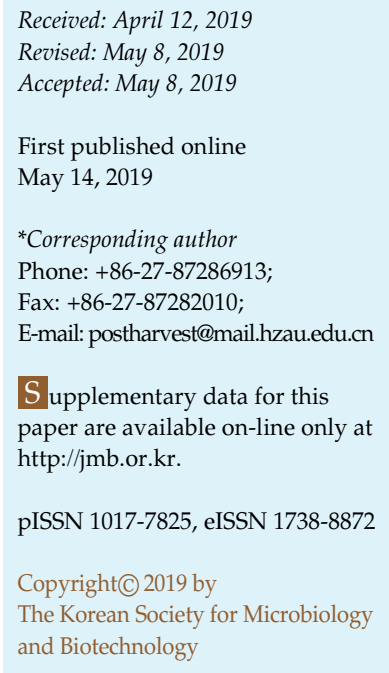

\begin{abstract}
Blue mold in citrus is caused by Penicillium italicum. In this study, the P. italicum-specific primers were developed for rapid detection based on the conserved genes RPB1 and RPB2 among Penicillium genomes. The two primer pairs RPB1-a and RPB1-b proved to be specific to detect $P$. italicum. The PCR assay among 39 fungal isolates and the colonial, pathogenic morphologies and molecular methods validated the specificity and reliability of these two primer pairs. This report provided a method and P. italicum-specific primers, which might greatly contribute to citrus postharvest industry.
\end{abstract}

Keywords: Penicillium italicum, specific primers, conserved gene, citrus
For species identification, it was a popular strategy to identify species by means of conserved genes (CG) and homologous alignments, and universal primers were used to perform PCR [1]. This method has been applied widely in animal, plant and microorganism identification [2-4]. However, species-level discrimination usually encountered highly homologous genes in closely-related species $[5,6]$. Universal primers might not be able to distinguish close species effectively, which often happened with the same genus [7]. With the environment becoming more complex, the variety and number of species increase more, especially in the microbial world. In citrus, a great variety of fungal pathogens cause postharvest diseases, which come from different genera, such as Penicillium, Aspergillus, Fusarium, Geotrichum, Alternaria, and Botrytis [8]. Some citrus Penicillium pathogens had high ITS (internal transcribed spacers) sequence identity. These all brought difficulty for species discrimination $[9,10]$. Therefore, a variety of different methods are encouraged and required to be developed for species identification.
Species-specific PCR was a good strategy to identify species [11]. This method was usually based on conserved genes for designing specific primers [12]. The genes RPB1 (RNA polymerase II largest subunit), RPB2 (RNA polymerase II second largest subunit), BTUB (beta tubulin), the mitochondrial cytochrome $c$ oxidase subunit I (COI) [13] and TEF (translation elongation factor 1 alpha) were often used [14]. The specific primers were generally designed based on the variable regions of the conserved genes among several inter-genus species [15]. In our previous study, the specific primers were developed to detect Penicillium digitatum rapidly among isolates in citrus [16].

Blue mold, caused by $P$. italicum, is a very serious postharvest disease in citrus [17]. This study designed $P$. italicum-specific primers targeting the RPB1 and RPB2 genes based on the genomes of $P$. italicum, $P$. digitatum, P. chrysogenum and P. expansum. Two primer pairs, RPB1-a and RPB1-b, were successfully developed by detecting $P$. italicum among citrus-related fungal isolates. P. italicum 
(B3), P. digitatum (N1), P. chrysogenum (Q) and P. expansum (L), which were deposited in Huazhong Agricultural University, China, were used as the sources of genomic DNA to develop the P. italicum-specific primers (Table S1). The gene sequences of RPB1 (Accession no. JN121649.1) and RPB2 (Accession no. KJ527377.1) of P. italicum were downloaded from the NCBI website (http://www.ncbi. nlm.nih.gov/) and subjected to BLAST analysis against the genomes of P. digitatum (Accession no. AKCU01000000), P. chrysogenum (JMSF01000000) and P. expansum (JQFZ01000000). The homology search produced the RPB1 and RPB2 sequences of each strain, which were used to design P. italicum-specific primers by using ClustalX 1.81 and Primer premier5. The cores of the primers were from the mutational sites in the homologous regions (Figs. 1, 2, and Table S2).

All the isolates used in this study were cultured using potato dextrose broth media (PDB) at $28^{\circ} \mathrm{C}$ for three days, respectively. The total DNA was extracted with a fungal genomic DNA extraction kit (Aidlab Biotech, China) and the concentrations were examined by using an ultra-micro UV spectrophotometer (Nanodrop 2000, Thermo Fisher Scientific). The DNA samples were diluted 20- and 100fold to reduce the amount of impurities (Table S1). PCR was performed in a $25 \mu \mathrm{l}$ reaction system $(1 \mu \mathrm{l} 10 \mu \mathrm{M}$ of each primer, $1 \mu \mathrm{l}$ DNA template, $12.5 \mu \mathrm{l} 2 \times \mathrm{Es}$ Taq

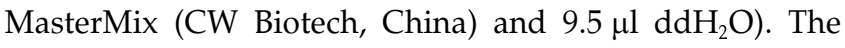
PCR condition was programed as follows: pre-heating at $94^{\circ} \mathrm{C}$ for $4 \mathrm{~min}, 30$ cycles of denaturation at $94^{\circ} \mathrm{C}$ for $30 \mathrm{sec}$, annealing at $64^{\circ} \mathrm{C}$ for $30 \mathrm{sec}$, and extension at $72^{\circ} \mathrm{C}$ for $45 \mathrm{sec}$, with a final extension at $72^{\circ} \mathrm{C}$ for $7 \mathrm{~min}$ [18].

The primers were tested for specificity in P. italicum (B3),

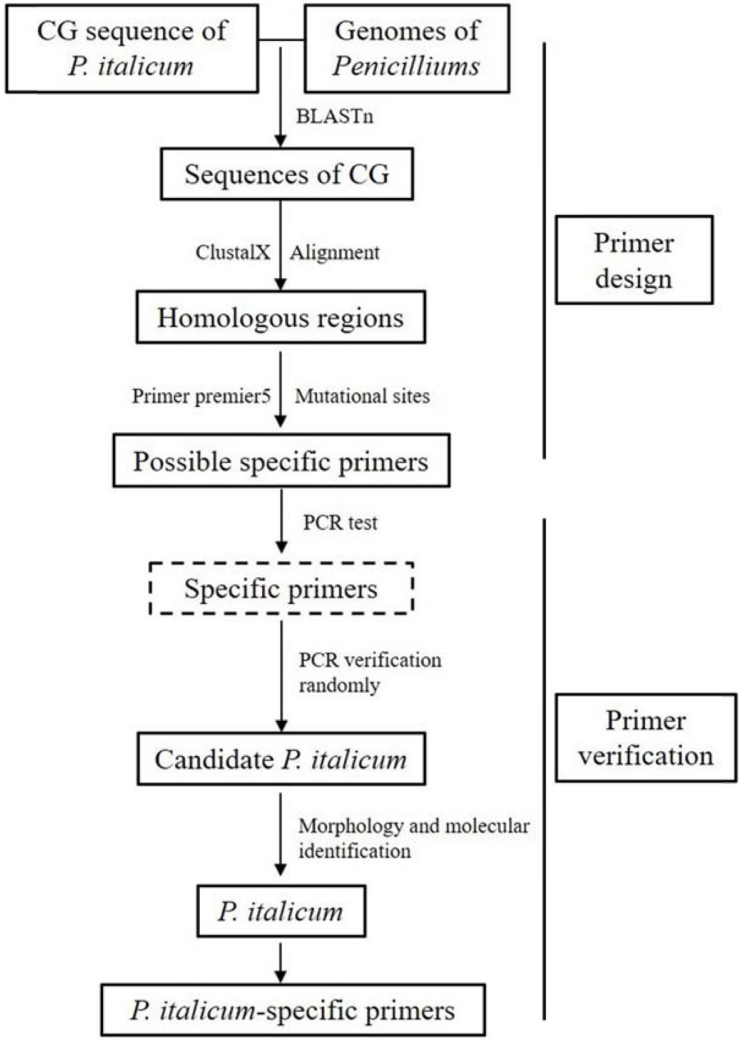

Fig. 1. The schema of the method for developing P. italicumspecific primers.

The image shows the whole process of the method, from primer designing to verifying. CG represents conserved genes.

P. digitatum (N1), P. chrysogenum (Q) and P. expansum (L) (Table S2). The results indicated that diluting the DNA templates 20-fold was not sufficient to exclude the

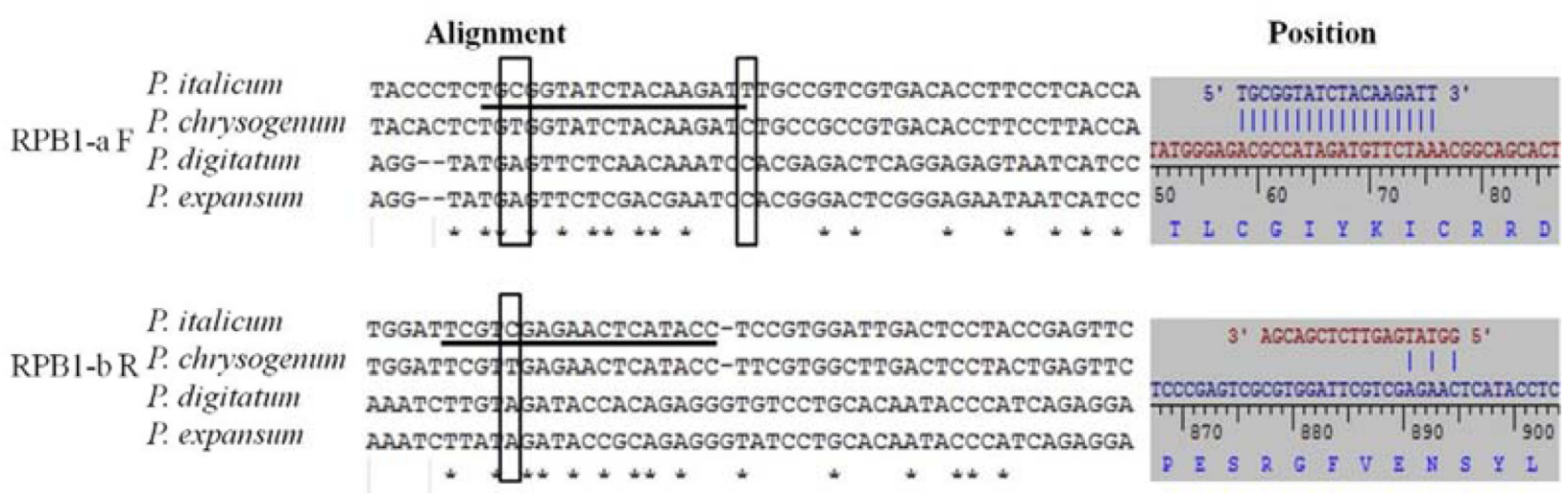

Fig. 2. Profile for designing the specific primers of P. italicum.

Sequences of the RPB1 genes of $P$. italicum, $P$. digitatum, P. chrysogenum and P. expansum were aligned using ClustalX, producing homologous regions. The mutational sites that showed that the nucleotide of $P$. italicum was different from the other three Penicilliums' were considered as primer positions. 
A

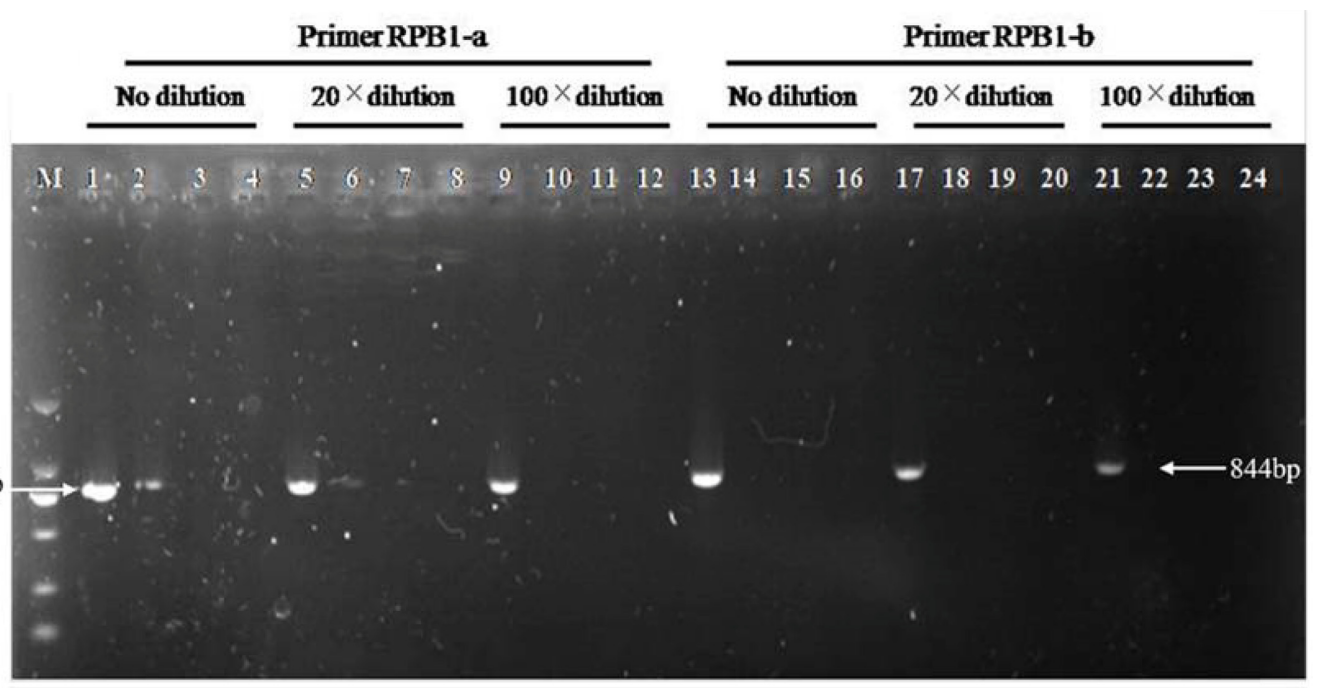

B

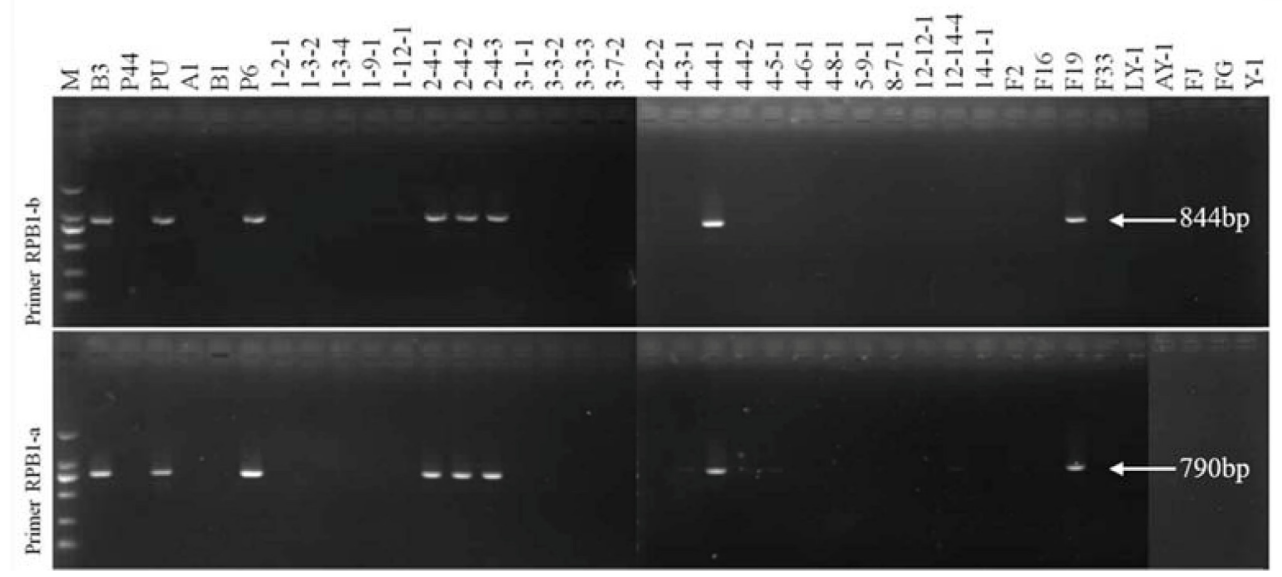

Fig. 3. Primer testing and candidate specific primer verification.

(A) Primer testing. Lane M, 2 kb molecular weight marker (Takara); lanes 1-4, P. italicum, P. digitatum, P. chrysogenum, P. expansum; lanes 5-8, 9-12, 13-16, 17-20, and 21-24, the same PCR templates as lanes 1-4. (B) Candidate specific primer verification by detecting P. italicum from 39 fungal isolates using the candidate primers RPB1-a and RPB1-b. Lane M, 2 kb molecular weight marker (Takara). The positions of the DNA bands were corresponding with their expected sizes.

interference of the impurities in the PCR (Fig. 3A). When diluting 100-fold, the effect of the impurity was absolutely excluded, and the blurry PCR band did not appear. Referring to the DNA concentrations in Table S1, diluting the DNA samples to $0.5-2 \mathrm{ng} / \mu \mathrm{l}$ might be suitable. The primer testing revealed that the primer pairs RBP1-a and RPB1-b were specific to detect P. italicum (Fig. 3A). The sequences of the PCR products also have high identity of 99\% with the organism P. italicum strain CBS 339.48 (RPB1, Accession No. JN121649.1) [19]. Therefore, these two primer pairs were regarded as candidate specific primers. To verify the accuracy and specificity of RPB1-a and RPB1$\mathrm{b}$, they were used to detect $P$. italicum among 39 fungal isolates (randomly isolated from citrus fruits), including some typical pathogens, such as Penicillium polonicum (A1), Penicillium crustosum (B1), Geotrichum citri-aurantii (AY-1), Alternaria solani (FJ), Rhizopus nigricans (FG) and Botrytis cinerea (Y-1) (Table S1). The DNA templates were directly diluted 100-fold. After PCR reaction, six unknown strains PU, 2-4-1, 2-4-2, 2-4-3, 4-4-1 and F19 were found out by the two primer pairs from the 39 fungal isolates. P. italicum (B3, P6) were recognized, P. digitatum (P44), P. polonicum (A1), P. crustosum (B1), G. citri-aurantii (AY-1), A. solani (FJ), R. nigricans $(\mathrm{FG}), B$. cinerea $(\mathrm{Y}-1)$ and the other strains could not be detected. In addition, RPB1-a and RPB1-b could offer the same detecting result and the PCR bands were all clear (Fig. 3B). This indicated that RPB1-a and RPB1-b were accurate and specific for detection of $P$. italicum. The six 

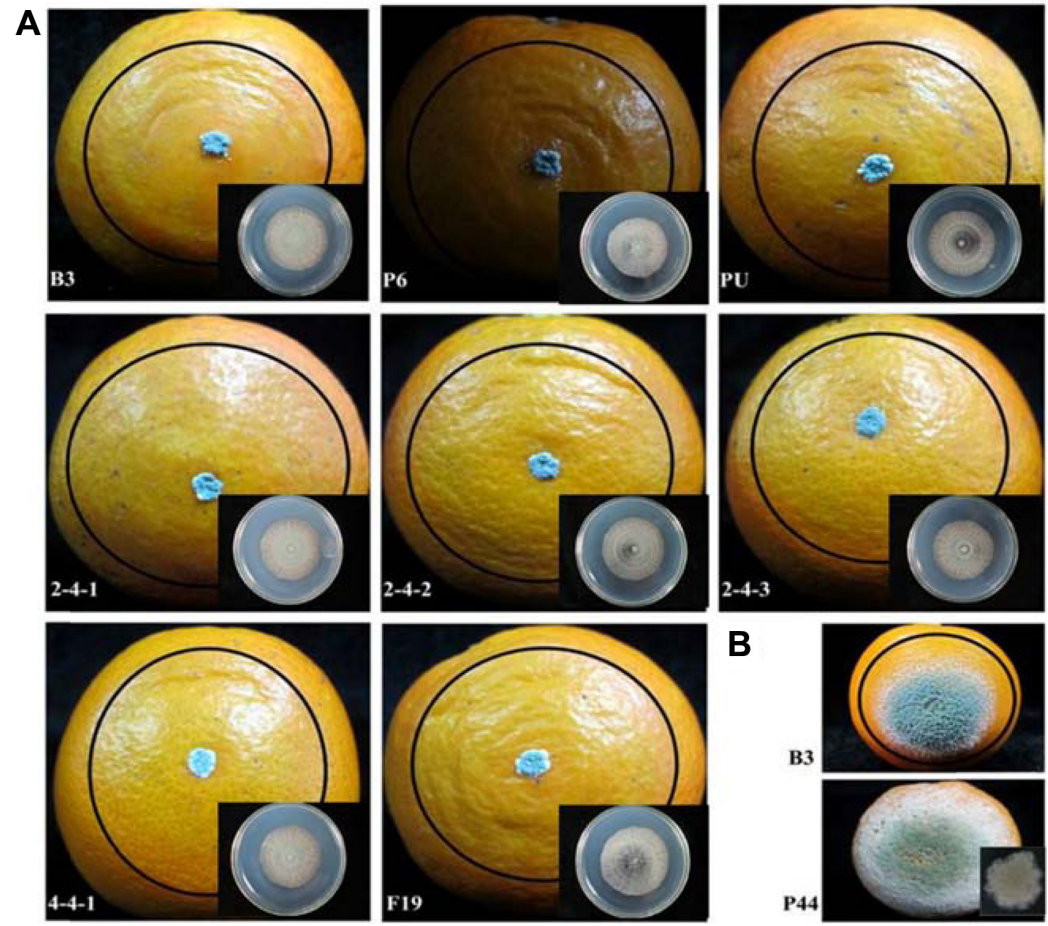

B

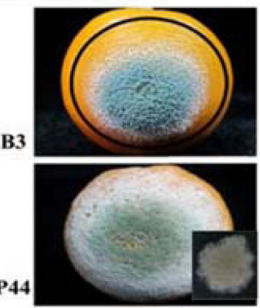

Fig. 4. Colonial and pathogenic morphologies of the candidate P. italicum strains.

(A) The colonial and pathogenic morphologies of the six candidate P. italicum strains and the control strains (P. italicum B3, P6) (cultivating on the fifth day). (B) The control groups P. italicum (B3) and P. digitatum (P44) on the sixth day.

strains could be taken as candidate P. italicum.

To determine if the candidate $P$. italicum strains were $P$. italicum, the six candidate $P$. italicum strains were observed for colonial morphology (inoculating $2 \mu \mathrm{l} 10^{5} \mathrm{CFU} / \mathrm{ml}$ spore suspension of each strain on the center of PDA media plates at $28^{\circ} \mathrm{C}$ for 6 days) and pathogenic morphology in citrus fruits. We pricked two wounds around the waist of "Fuben" navel orange fruits and inoculated $10 \mu 110^{6} \mathrm{CFU} / \mathrm{ml}$ spore suspension of each strain in the wounds. All of the in vitro or in vivo experiments, as well as the morphologies of the six candidate $P$. italicum strains were basically consistent with P.italicum (B3, P6) (Fig. 4). The navel orange fruits turned seriously watery around the wounds on the fifth day, with a mass of spores germinating in the wounds (Fig. 4A). On the sixth day, the mycelia and spores spread around fast (Fig. 4B) [20], and the pathogenic morphology was greatly different from $P$. digitatum. In addition, the six candidate $P$. italicum strains were examined by molecular identification with the primer pair ITS1 5'TCCG TAGG TGAA CCTG CGG-3', ITS4 5'-TCCT CCGC TTAT TGAT ATGC-3'. The result also indicated the ITS sequences of the six candidate $P$. italicum strains had high identity of $99 \%$ with P. italicum (Table S3) [21]. These results revealed that the candidate $P$. italicum strains PU, 24-1, 2-4-2, 2-4-3, 4-4-1, and F19 were exactly P. italicum, which demonstrated that the two pairs of primers, RPB1-a and RPB1-b, were accurate and specific for P. italicum detection. They could be used to detect $P$. italicum rapidly among a large number of unknown citrus postharvest pathogens. This research may significantly facilitate the fungal detection in the citrus industry, and this method may also be utilized as a reference for species detection in other fields.

\section{Acknowledgments}

This study was funded by the National Natural Science Foundation of China (grant no. 31672205), the earmarked fund for China Agriculture Research System CARS-26 and the National Key Research and Development Program (Program no. 2016YFD0400904).

\section{Conflict of Interest}

The authors have no financial conflicts of interest to declare. 


\section{References}

1. Schoch CL, Seifert KA, Huhndorf S, Robert V, Spouge JL, Levesque CA, et al. 2012. Nuclear ribosomal internal transcribed spacer (ITS) region as a universal DNA barcode marker for Fungi. Proc. Natl. Acad. Sci. USA 109: 6241-6246.

2. Parson W, Pegoraro $K$, Niederstätter $H$, Föger $M$, Steinlechner M. 2000. Species identification by means of the cytochrome b gene. Int. J. Legal Med. 114: 23-28.

3. Chen S, Yao H, Han J, Liu C, Song J, Shi L, et al. 2010. Validation of the ITS2 region as a novel DNA barcode for identifying medicinal plant species. PLoS One 5: e8613.

4. Kiss L. 2012. Limits of nuclear ribosomal DNA internal transcribed spacer (ITS) sequences as species barcodes for Fungi. Proc. Natl. Acad. Sci. USA 109: E1811.

5. Fox GE, Wisotzkey JD, Jurtshuk JR P. 1992. How close is close: $16 \mathrm{~S}$ rRNA sequence identity may not be sufficient to guarantee species identity. Int. J. Syst. Evol. Microbiol. 42: 166-170.

6. Song YL, Kato N, Liu CX, Matsumiya Y, Kato H, Watanabe K. 2000. Rapid identification of 11 human intestinal Lactobacillus species by multiplex PCR assays using group-and speciesspecific primers derived from the 16S-23S rRNA intergenic spacer region and its flanking $23 \mathrm{~S}$ rRNA. FEMS Microbiol. Lett. 187: 167-173.

7. Rikkinen J. 2013. Molecular studies on cyanobacterial diversity in lichen symbioses. MycoKeys 6: 3-32.

8. Tournas V, Katsoudas E. 2005. Mould and yeast flora in fresh berries, grapes and citrus fruits. Int. J. Food Microbiol. 105: $11-17$

9. Wiese J, Ohlendorf B, Blümel M, Schmaljohann R, Imhoff JF. 2011. Phylogenetic identification of fungi isolated from the marine sponge Tethya aurantium and identification of their secondary metabolites. Mar. Drugs 9: 561-585.

10. Xu X, Chen J, Xu H, Li D. 2014. Role of a major facilitator superfamily transporter in adaptation capacity of Penicillium funiculosum under extreme acidic stress. Fungal Genet. Biol. 69: 75-83.

11. Zhu P, Wu L, Liu L, Huang L, Wang Y, Tang W, et al. 2013. Fusarium asiaticum: an emerging pathogen jeopardizing postharvest asparagus spears. J. Phytopathol. 161: 696-703.

12. Martineau F, Picard FJ, Ke D, Paradis S, Roy PH, Ouellette M, et al. 2001. Development of a PCR assay for identification of staphylococci at genus and species levels. J. Clin. Microbiol. 39: 2541-2547.

13. Alastruey-Izquierdo A, Hoffmann K, de Hoog GS, Rodriguez-Tudela JL, Voigt K, Bibashi E, et al. 2010. Species recognition and clinical relevance of the zygomycetous genus Lichtheimia (syn. Absidia pro parte, Mycocladus). J. Clin. Microbiol. 48: 2154-2170.

14. Kepler RM, Humber RA, Bischoff JF, Rehner SA. 2014. Clarification of generic and species boundaries for Metarhizium and related fungi through multigene phylogenetics. Mycologia 106: $811-829$.

15. Walter J, Tannock GW, Tilsala-Timisjarvi A, Rodtong S, Loach DM, Munro K, et al. 2000. Detection and identification of gastrointestinal Lactobacillus species by using denaturing gradient gel electrophoresis and species-specific PCR primers. Appl. Environ. Microbiol. 66: 297-303.

16. Chen K, Tian Z, Wang L, Long CA. 2017. Development of specific primers based on the genomes of Penicillium spp. for rapid detection of Penicillium digitatum among fungal isolates in citrus. Eur. J. Plant Pathol. 149: 201-209.

17. Youssef K, Ligorio A, Sanzani SM, Nigro F, Ippolito A. 2012. Postharvest Biol. Technol. 72: 57-63.

18. Yin GH, Zhang YL, Pennerman KK, Wu GX, Hua SST, Yu JJ, et al. 2017. Characterization of blue mold Penicillium species isolated from stored fruits using multiple highly conserved loci. J. Fungi 3: 12.

19. McGinnis S, Madden TL. 2004. BLAST: at the core of a powerful and diverse set of sequence analysis tools. Nucleic Acids Res. 32: 20-25.

20. Li BQ, Zong Y, Du Z, Chen Y, Zhang Z, Qin GZ, et al. 2015 Genomic characterization reveals insights into patulin biosynthesis and pathogenicity in Penicillium species. Mol. Plant Microbe Interact. 28: 635-647.

21. Nilsson RH, Kristiansson E, Ryberg M, Hallenberg N, Larsson KH. 2008. Intraspecific ITS variability in the kingdom Fungi as expressed in the international sequence databases and its implications for molecular species identification. Evol. Bioinform. Online 4: 193-201.

22. Marcet-Houben M, Ballester AR, de la Fuente B, Harries E, Marcos JF, González-Candelas L, et al. 2012. Genome sequence of the necrotrophic fungus Penicillium digitatum, the main postharvest pathogen of citrus. BMC Genomics 13: 646.

23. Specht T, Dahlmann TA, Zadra I, Kürnsteiner H, Kück U. 2014. Complete sequencing and chromosome-scale genome assembly of the industrial progenitor strain P2niaD18 from the penicillin producer Penicillium chrysogenum. Genome Announc. 2: e00577-14.

24. Amselem J, Cuomo CA, Van Kan JA, Viaud M, Benito EP, Couloux A, et al. 2011. Genomic analysis of the necrotrophic fungal pathogens Sclerotinia sclerotiorum and Botrytis cinerea. PLoS Genet. 7: e1002230. 